\title{
An experimental investigation on thermal properties of molybdenum disulfide nanofluids
}

\author{
Yu Su, a, Le Gong ${ }^{1, b}$, Bi Li ${ }^{1, c}$, Dandan Chen ${ }^{1, d}$ \\ ${ }^{1}$ College of Mechanical Engineering, Jiangsu University of Science and Technology, Zhenjiang \\ 212003, China \\ asuyuliu@sohu.com, bgongle2009@163.com, '58863024@qq.com, d1264923189@qq.com
}

Keywords: Molybdenum disulfide; Nanofluids; Suspension stability; Thermal conductivity; Surface tension

\begin{abstract}
This paper prepared $\mathrm{MoS}_{2}$ water-based and oil-based nanofluids with different mass fractions by two step method, and investigated their stability, thermal conductivity, and surface tension. The thermal conductivity of $\mathrm{MoS}_{2}$ oil-based nanofluids was calculated using the classic model, and compared with experimental result. The results indicate that the stability for $\mathrm{MoS}_{2}$-LB2000 oil nanofluid with a mass fraction of 0.01 wt. $\%$ was better than that for $\mathrm{MoS}_{2}$-LB2000 oil nanofluid with a mass fraction of $0.05 \mathrm{wt} \%$. Compared with base fluids, $\mathrm{MoS}_{2}$ water-based and oil-based nanofluids had higher thermal conductivity and lower surface tension. With the increase of mass fraction, the thermal conductivity and surface tension of $\mathrm{MoS}_{2}$ nanofluids increased and decreased, respectively. The experimental results for the thermal conductivity of $\mathrm{MoS}_{2}$ oil-based nanofluids was higher than their theoretical predictions.
\end{abstract}

\section{Introduction}

Nanofluids are suspensions obtained by suspending nanoparticles with average sizes below $100 \mathrm{~nm}$ in base fluids. Since solids conduct heat better than liquids, the dispersed nanoparticles enhance the thermal conductivity of base fluid. A small amount of nanoparticles, when dispersed uniformly and suspended stably in base fluids, can provide exaggerated improvements in the thermal properties of base fluids [1]. It is well known that during the mechanical process, the friction between tool and workpiece generates a lot of heat, which causes the rapid tool wear and decreases the surface quality. Due to the enhanced thermal properties and lubrication abilities, it appears to be a promising approach to use the nanofluids as cutting fluids to reduce the machining temperature.

Over the past decade, the most common nanoparticles, studied in the field of thermal nanofluids engineering, are copper $(\mathrm{Cu})$, aluminium oxide $\left(\mathrm{Al}_{2} \mathrm{O}_{3}\right)$, and carbon nanotubes $(\mathrm{CNTs})$. From experimental results, Kole et al. [2] reported that the thermal conductivity of Cu-gear oil nanofluid increases non-linearly with nanoparticles concentration and shows a maximum enhancement of $24 \%$ for nanofluid containing 2 vol. \% Cu nanoparticles in gear oil. Syam Sundar et al. [3] studied the thermal conductivity of nanofluids by dispersing $\mathrm{Al}_{2} \mathrm{O}_{3}$ nanoparticles in water and ethylene glycol at different temperature and volume concentrations, and reported that the maximum thermal conductivity enhancement reaches up to $32.26 \%$ for the nanofluid containing 1.5 vol. $\% \mathrm{Al}_{2} \mathrm{O}_{3}$ at $60^{\circ} \mathrm{C}$. Kumaresan et al. [4] reported the largest magnitude of thermal conductivity enhancement about $19.73 \%$, when 0.45 vol. \% CNTs was added in water and ethylene glycol at $40^{\circ} \mathrm{C}$.

Although the nanofluid has many advantages, the stability problem has not obtained very good solution, which has become an obstacle for nanofluids in the field of engineering application. So it is very necessary to study the stability of the suspension. At the same time, many researchers focus on the viscosity, thermal conductivity and other thermal properties of nanofluids in recent years. However, the research reports on the surface tension of the nanofluids are very few. Therefore this paper first adopted two step method to prepare molybdenum disulfide nanofluids, and investigated the effect of mass fraction on the suspension stability. Then TC3010L thermal conductivity meter 
was used to measure the thermal conductivity of molybdenum disulfide nanofluids, and the experimental results and theoretical predictions were compared. Finally, automatic surface tension meter was used to measure the surface tension of molybdenum disulfide nanofluids, and the influence of mass fraction on surface tension of molybdenum disulfide nanofluids was studied.

\section{Experimental details}

The base fluids used in this research involve deionized water, LB2000 vegetable lubricants, and PriECO6000 unsaturated polyhydric alcohol ester. Molybdenum disulfide nanoparticles, purchased from Nanjing GY Co., Ltd. with the diameters of $30 \mathrm{~nm}$, were used throughout the experiments. The molybdenum disulfide water-based and oil-based nanofluids with different mass fractions ranging from 0 to 0.5 wt. \% were prepared by dispersing a certain amount of molybdenum disulfide nanoparticles in base fluids using KQ-100DE ultrasonic cleaner with a $100 \mathrm{~W}$ output power and 40 KHZ frequency. The ultrasonication time used was two hours for all of the nanofluids prepared.

A U-4100 UV-visible spectrophotometer was employed to measure the absorbance of $\mathrm{MoS}_{2}$-LB2000 nanofluids at regular intervals after preparation, and evaluate the suspension stability. A BZY-1 automatic surface tension meter, which is produced by Shanghai Precision Instrument Co., Ltd., was used to measure the surface tension of molybdenum disulfide nanofluids. TC3010L thermal conductivity measuring instrument, which is produced based on the transient hot-wire method by Xi' an Xiatech Electronic Technology Co. Ltd., was used to measure the thermal conductivity of nanofluids prepared. The measurement range of TC3010L is $0.001-5 \mathrm{~W} / \mathrm{Km}$. All of the measurements were taken three times at room temperature and the average of three values was used for analysis.

\section{Results and discussion}

Suspension stability. As the nanoparticles have a huge specific surface area and high surface energy, they are prone to agglomerate, thus affecting the performance of nanoparticles. So the study of dispersion stability of nanofluids is a prerequisite for the application. Figs. 1 and 2 show the UV Vis absorption spectrum and absorbance of $\mathrm{MoS}_{2}-\mathrm{LB} 2000$ nanofluids with different mass fractions for different days after preparation, respectively. It can be seen from Fig. 1 that there is an obvious peak at the wavelength of 600 and $650 \mathrm{~nm}$, respectively. According to the literature, UV absorption peak of $\mathrm{MoS}_{2}$ lies between 620 and $670 \mathrm{~nm}$. So the absorption peak at $650 \mathrm{~nm}$ was used to analyze the stability of suspension. As shown in Figs. 1 and 2, the higher the concentration, the higher the absorption peak. This can be attributed to more nanoparticles suspended in LB2000 vegetable lubricants when increasing mass fraction. With the increase of standing time, the peak value decreased, indicating that some nanoparticles deposited. Furthermore, the reduction in peak value for $\mathrm{MoS}_{2}$-LB2000 nanofluid with the mass fraction of $0.05 \mathrm{wt}$. \% was more than that for $\mathrm{MoS}_{2}$-LB2000 nanofluid with the mass fraction of 0.01 wt. \%. This indicates that the stability of the latter was better than the former.

Influence of mass fraction on thermal conductivity. The factors affecting the thermal conductivity of nanofluids include mass fraction of nanoparticles, type, size, shape, temperature, etc. However, a lot of research show that the most significant factor for the thermal conductivity of nanofluids is the mass fraction of nanoparticles. Therefore this paper focuses on the effect of mass fraction on thermal conductivity of $\mathrm{MoS}_{2}$ nanofluids. Figs. 3 and 4 show the variation of thermal conductivity of $\mathrm{MoS}_{2}$ water-based and oil-based nanofluids with mass fraction, respectively. As shown in Figs. 3 and 4, compared with base fluids, $\mathrm{MoS}_{2}$ water-based and oil-based nanofluids had higher thermal conductivity. Besides it, with the increase of mass fraction, the thermal conductivity of $\mathrm{MoS}_{2}$ water-based and oil-based nanofluids increased. This is because the larger mass fraction, the more nanoparticles per unit mass fraction of the suspension, thus causing the heat transfer enhancement between particles, and the micro convection enhancement between particle and base fluid. The enhancement in thermal conductivity for $\mathrm{MoS}_{2}$ water-based nanofluids seemed higher 
than that for $\mathrm{MoS}_{2}$ oil-based nanofluids. Furthermore, at the same mass fraction, the increase in thermal conductivity for $\mathrm{MoS}_{2}$-PriECO6000 nanofluid was more than that for $\mathrm{MoS}_{2}-\mathrm{LB} 2000$ nanofluid. The reason for this phenomenon is that better dispersion and suspension stability was achieved when using PriECO6000 as base oil.

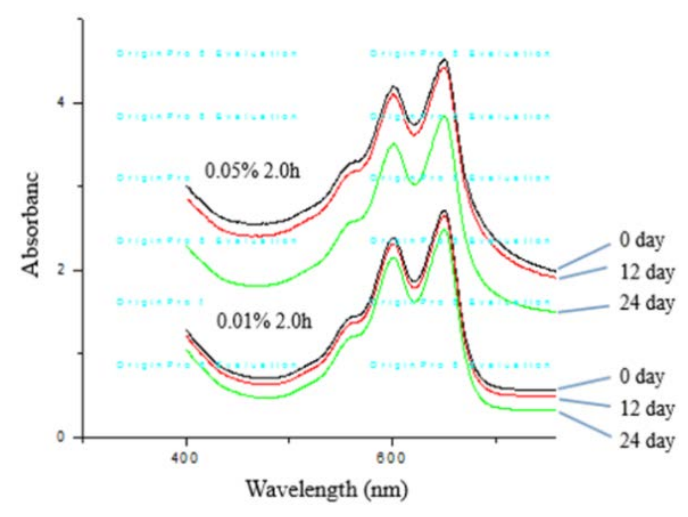

Fig. 1. UV-visible absorption spectrum of molybdenum disulfide nanofluids

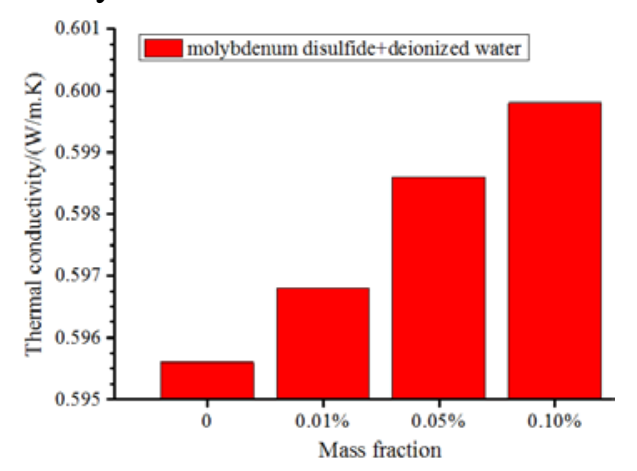

Fig. 3. Effect of mass fraction on the thermal conductivity of water-based nanofluids

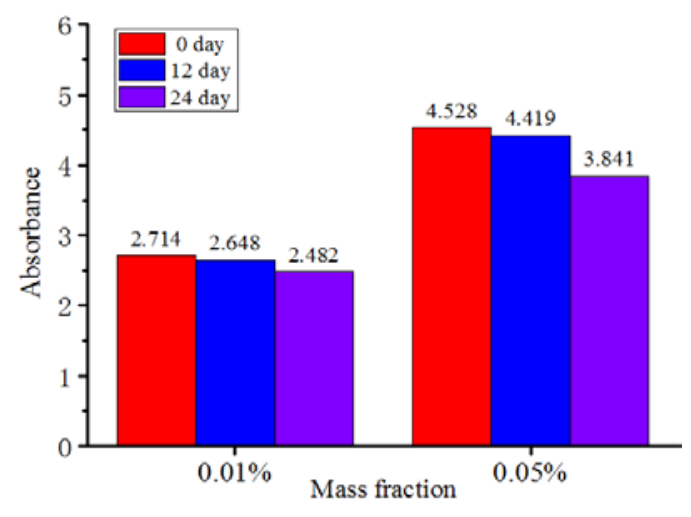

Fig. 2. UV-visible absorbance of molybdenum disulfide nanofluids

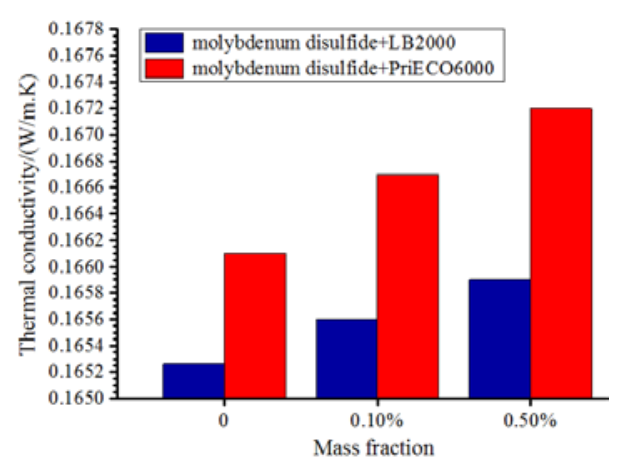

Fig. 4. Effect of mass fraction on the thermal conductivity of oil-based nanofluids

Comparison of experimental results with theoretical predictions. Thermal conductivity is the main thermal properties of nanofluids. However, the calculation of thermal conductivity has not yet been established for all expressions of nanofluids. Table 1 show the calculation model of thermal conductivity of nanofluids.

Table 1 Thermal conductivity models

\begin{tabular}{|c|c|}
\hline Models & Expression \\
\hline \multirow{2}{*}{ Maxwell model [5] } & $K_{\text {eff }} \quad K_{\mathrm{p}}+2 K_{\mathrm{f}}+2\left(K_{\mathrm{p}}-K_{\mathrm{f}}\right) \phi$ \\
\hline & $\overline{K_{\mathrm{f}}}=\overline{K_{\mathrm{p}}+2 K_{\mathrm{f}}-\left(K_{\mathrm{p}}-K_{\mathrm{f}}\right) \phi}$ \\
\hline \multirow{2}{*}{ Tim model [6] } & $\underline{K_{\mathrm{nf}}}=\left[1+\underline{3\left(K_{\mathrm{p}}-K_{\mathrm{bf}}\right) \phi}\right]$ \\
\hline & $\overline{K_{\mathrm{bf}}}-\left[1+\overline{\left(K_{\mathrm{p}}+2 K_{\mathrm{bf}}\right)}\right]$ \\
\hline \multirow[b]{2}{*}{$\begin{array}{c}\text { Hamilton and Crosser } \\
\text { model [7] }\end{array}$} & $K_{\text {eff }}-K_{\mathrm{p}}+(\mathrm{n}-1) K_{\mathrm{f}}-(\mathrm{n}-1)\left(K_{\mathrm{p}}-K_{\mathrm{f}}\right) \phi$ \\
\hline & $\begin{array}{c}K_{\mathrm{f}} \\
\text { where } \mathrm{n}=3 \text { for spherical particles and } \mathrm{n}=6 \text { for cylindrical } \\
\text { particles }\end{array}$ \\
\hline \multirow{2}{*}{ Wasp model [8] } & $K_{\mathrm{nf}}=\left[K_{\mathrm{p}}+2 K_{\mathrm{bf}}-2 \phi\left(K_{\mathrm{bf}}-K_{\mathrm{p}}\right)\right.$ \\
\hline & $K_{\mathrm{bf}}\left[K_{\mathrm{p}}+2 K_{\mathrm{bf}}+2 K_{\mathrm{p}}\left(K_{\mathrm{bf}}-K_{\mathrm{p}}\right)\right]$ \\
\hline
\end{tabular}


The classic Maxwell model and Tim model were used for calculating the predicted value of thermal conductivity. Then, experimental results of thermal conductivity were compared with its theoretical predictions, as shown in Fig. 5. Curves A1, A2, and A3 represent the experimental result, predicted value using Maxwell model and Tim model of thermal conductivity for $\mathrm{MoS}_{2}$-LB2000 nanofluid, respectively. Curves B1, B2, and B3 represent the experimental result, predicted value using Maxwell model and Tim model of thermal conductivity for $\mathrm{MoS}_{2}$-PriECO6000 nanofluid, respectively. It can be seen from Fig. 5 that the predicted value of thermal conductivity are always lower than its experimental result, irrespective of type of base fluid. This indicates that the calculated values of thermal conductivity using these theoretical models cannot correctly reflect the actual thermal conductivity of nanofluids.

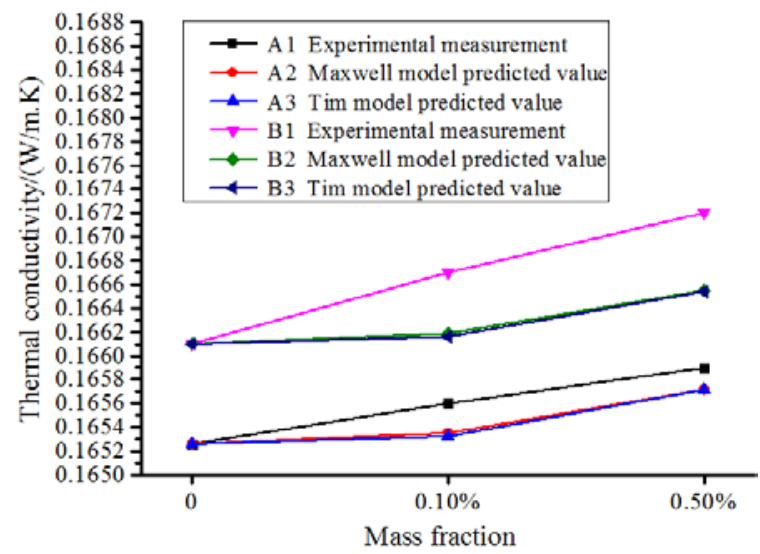

Fig. 5. Comparison of experimental results of thermal conductivity with its theoretical predictions

Surface tension. Fig. 6 shows the variation of surface tension with mass fraction for $\mathrm{MoS}_{2}$ oilbased nanofluids. As shown in Fig. 6, the surface tension of $\mathrm{MoS}_{2}-\mathrm{LB}_{2000}$ and $\mathrm{MoS}_{2}$-PriECO6000 nanofluids was lower than that of LB2000 and PriECO6000, respectively. This may be due to the fact that nanoparticles automatically aggregated to the surface of base oil. With the increase of mass fraction of nanoparticles, the surface tension of these two $\mathrm{MoS}_{2}$ oil-based nanofluids gradually reduced. This is because the concentration of nanoparticles in the surface layer increased.

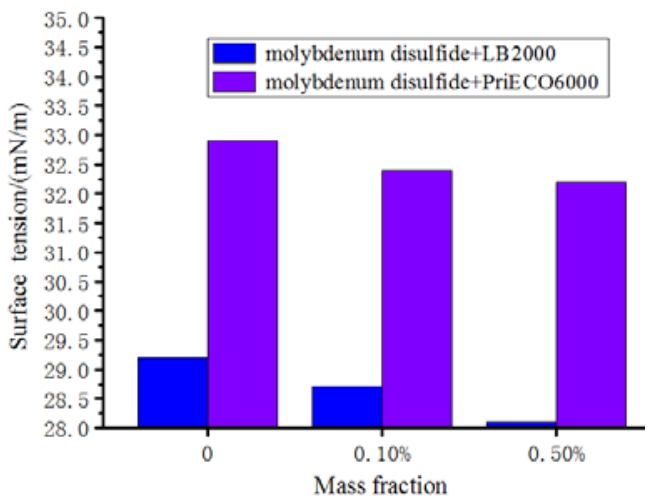

Fig. 6. Effect of mass fraction on the surface tension of oil-based nanofluids

\section{Conclusion}

Based on the results of experimental investigation, the following conclusions can be drawn:

1) The absorbance of $\mathrm{MoS}_{2}$-LB2000 nanofluids was measured to evaluate the stability. With the increase of standing time, the reduction in value of absorption peak for $\mathrm{MoS}_{2}$-LB2000 nanofluid with the mass fraction of 0.05 wt. \% was more than that for $\mathrm{MoS}_{2}$-LB2000 nanofluid with the mass fraction of $0.01 \mathrm{wt}$ \%, indicating that the stability of the former was worse than that of the latter.

2) $\mathrm{MoS}_{2}$ water-based and oil-based nanofluids had higher thermal conductivity than base fluids. With the increase of mass fraction of nanoparticles, the thermal conductivity increased. The enhancement in thermal conductivity for $\mathrm{MoS}_{2}$ water-based nanofluids was higher than that for 
$\mathrm{MoS}_{2}$ oil-based nanofluids. At the same mass fraction, $\mathrm{MoS}_{2}$-PriECO6000 nanofluids had more increase in thermal conductivity compared with $\mathrm{MoS}_{2}-\mathrm{LB} 2000$ nanofluids The predicted thermal conductivity for $\mathrm{MoS}_{2}$ oil-based nanofluids was lower than the corresponding experimental result.

3) The surface tension of $\mathrm{MoS}_{2}$ oil-based nanofluids was lower than their base oils. In addition, with the increase of mass fraction, the surface tension of $\mathrm{MoS}_{2}$ oil-based nanofluids decreased.

\section{Acknowledgements}

The author wishes to acknowledge with financial support of this research by National Natural Science Foundation of China under contract no. 51205177, Natural Science Foundation of Jiangsu Province under contract no. BK2012277, Natural Science Program for Basic Research of Jiangsu Province under contract no. 08KJB460002, Qing Lan Project, and Research Fund of DML-HYIT (HGDML-0901).

\section{References}

[1] S.K. Das, U.S. Stephen, U.S. Choi, et al. Nanofluids—science and technology [C]. USA: John Wiley and Sons, Inc. 2008.

[2] M. Kole, T.K. Dey. Enhanced thermophysical properties of copper nanoparticles dispersed in gear oil [J]. Applied Thermal Engineering, 2013, 56(1-2): 45-53.

[3] L. Syam Sundar, E. Venkata Ramana, Manoj K. Singh, et al. Thermal conductivity and viscosity of stabilized ethylene glycol and water mixture $\mathrm{Al}_{2} \mathrm{O}_{3}$ nanofluids for heat transfer applications: An experimental study [J]. International Communications in Heat and Mass Transfer, 2014, 56: 86-95.

[4] V. Kumaresan, R. Velraj. Experimental investigation of the thermo-physical properties of water-ethylene glycol mixture based CNT nanofluids [J]. Thermochimica Acta, 2012, 545: 180186.

[5] J.C. Maxwell. A treatise on Electricity and Magnetism. Oxford, Univ. Press, Cambridge, 1904.

[6] E.V. Timofeeva, A.N. Gavrilov, J.M. McCloskey, et al. Thermal conductivity and particle agglomeration in alumina nanofluids, experiment and theory [J]. Phys Rev E, 2007, 76: 1-16.

[7] R.L. Hamilton, O.K. Crosser. Thermal conductivity of heterogeneous two-component systems [J]. Ind Eng Chem Fundam, 1962, 1(3):187-191.

[8] F.J. Wasp. Solid-liquid slurry pipeline transportation [C]. Berlin: Trans Tech Publications, 1977. 